\title{
atlas.brussels, un outil de géovisualisation de l'extension et de la fragmentation métropolitaine bruxelloise
}

Olivier Finance, Arnaud Adam et Isabelle Thomas

\section{(apenEdition}

\section{Édition électronique}

URL : https://journals.openedition.org/mappemonde/5506

DOI : 10.4000/mappemonde.5506

ISSN : 1769-7298

Éditeur

UMR ESPACE

Référence électronique

Olivier Finance, Arnaud Adam et Isabelle Thomas, « atlas.brussels, un outil de géovisualisation de l'extension et de la fragmentation métropolitaine bruxelloise », Mappemonde [En ligne], 130 | 2021, mis en ligne le 15 mars 2021, consulté le 20 mai 2021. URL : http://journals.openedition.org/ mappemonde/5506 ; DOI : https://doi.org/10.4000/mappemonde.5506

Ce document a été généré automatiquement le 20 mai 2021.

\section{(i) (2)}

La revue Mappemonde est mise à disposition selon les termes de la Licence Creative Commons Attribution - Pas d'Utilisation Commerciale - Partage dans les Mêmes Conditions 4.0 International. 


\title{
atlas.brussels, un outil de géovisualisation de l'extension et de la fragmentation métropolitaine bruxelloise
}

\author{
Olivier Finance, Arnaud Adam et Isabelle Thomas
}

URL du site : https://atlas.brussels/

\section{Introduction}

Bruxelles constitue de longue date un objet d'étude singulier dans la littérature sur les villes européennes, du fait de ses particularités politiques et administratives, sociales, linguistiques ou encore économiques. Des travaux ont abordé l'extension spatiale de son agglomération et de son aire métropolitaine, cette dernière s'étendant sur chacune des trois Régions belges (Van Hecke, 1999; Thomas et al., 2012; Jones et al., 2015; Dujardin et al., 2007; Vanderstraeten et Van Hecke, 2019; Strale, 2019); aucun consensus quant à son extension exacte ne se dégage. À l'image de la Belgique tout entière (Van Wynsberghe, 2011; Godin et Hindriks, 2015), l'aire métropolitaine de Bruxelles présente une certaine fragmentation, et ce dans diverses dimensions (Van Wynsberghe et al., 2009). Analyser cette fragmentation a été un des objectifs du projet de recherche «Bru-Net » abordant de manière originale la métropole bruxelloise sous l'angle des réseaux (Bruxelles dans les réseaux et les réseaux dans Bruxelles) plutôt qu'en se contentant de caractéristiques attributaires propres à chaque unité spatiale. L'intensité variable des liens qui unissent des couples d'unités spatiales a permis de révéler des bassins d'interactions préférentielles internes à la métropole bruxelloise qui témoignent de cette relative fragmentation, à partir de nombreux réseaux spatialisés d'interactions: des mobilités de personnes, des relations interpersonnelles ou encore des relations économiques. Ainsi, l'objectif de ce projet a 
été d'analyser comment Bruxelles se détache comme une entité spécifique, et éventuellement cohérente, dans ces réseaux à l'échelle nationale (délimitation de l'aire métropolitaine), et d'analyser, au niveau métropolitain, sa fragmentation interne au regard de ces mêmes réseaux.

2 L'objectif de cet article est de présenter, au-delà des questionnements généraux du projet de recherche, un outil de géovisualisation en ligne des résultats élaborés dans ce cadre : atlas.brussels. Cette plateforme vise à rassembler, compléter, étendre et valoriser les divers résultats produits au cours du projet, en complément des articles scientifiques publiés et d'une thèse de doctorat (Adam, 2019). Cet outil permet, avant tout, de rendre compte de la diversité des configurations spatiales des bassins d'interactions préférentielles détectés selon la nature des relations mesurées, selon l'échelle (nationale ou métropolitaine) et selon les spécifications de l'algorithme qui permet de les détecter. L'outil permet aussi d'explorer visuellement les relations éventuelles entre les configurations spatiales de ces bassins et d'autres dimensions de différenciation de l'espace.

Le site se compose d'une page principale comportant l'outil de géovisualisation et d'un ensemble de pages annexes détaillant davantage les jeux de données, les objectifs généraux du projet, ou encore les publications réalisées dans le cadre du projet. Dans la suite de cette contribution, atlas.brussels fera référence à la seule page comportant la géovisualisation. L'atlas est présenté dans une première section. Par la suite, sont détaillés la méthode retenue pour comparer les réseaux d'interaction les uns par rapport aux autres et les différents objectifs initiaux de la géovisualisation qui ont des implications dans la sémiologie graphique et les solutions techniques retenues. Les aspects techniques de la réalisation d'atlas.brussels sont enfin développés avant une brève conclusion.

\section{Présentation générale de l'atlas}

4 atlas.brussels rassemble dans une interface unique plus d'une centaine de cartes avec pour originalité, lorsque les données le permettent, de les proposer à deux échelles différentes (nationale et métropolitaine) et à diverses granularités (communes, secteurs statistiques - équivalent des IRIS en France -, maillage régulier, etc.). Des cartes dites de contextualisation peuvent premièrement être affichées, présentant des propriétés des unités spatiales en elles-mêmes (par exemple les figures 1a à 1c: la densité de population, la proportion de surface bâtie, ou encore l'occupation du sol dans l'ancienne province du Brabant, considérée ici comme une délimitation grossière de l'hinterland bruxellois. Des cartes certes plus complexes dans leur réalisation, mais très parlantes visuellement, peuvent également être affichées: il s'agit de cartes chorochromatiques qui illustrent des bassins d'interactions préférentielles. Concrètement, il s'agit de faire émerger des étendues spatiales, territoriales, à l'intérieur desquelles les échanges sont plus intenses qu'entre ces différentes étendues. Ces bassins d'interactions préférentielles sont donc construits à partir d'un algorithme de détection de communautés (voir section suivante) appliqué à des matrices originedestination résumant divers types de réseaux (par exemple les figures $1 \mathbf{d}$ et $1 \mathbf{e}$ : bassins d'interactions préférentielles construits à partir des déplacements de camions et des appels téléphoniques). Dans certains cas, atlas.brussels permet la superposition de cartes de contextualisation et des bassins d'interactions préférentielles (figure 1f). 
Figure 1. Six exemples de cartes pouvant être obtenues dans l'outil atlas.brussels à l'échelle de l'ancienne province de Brabant

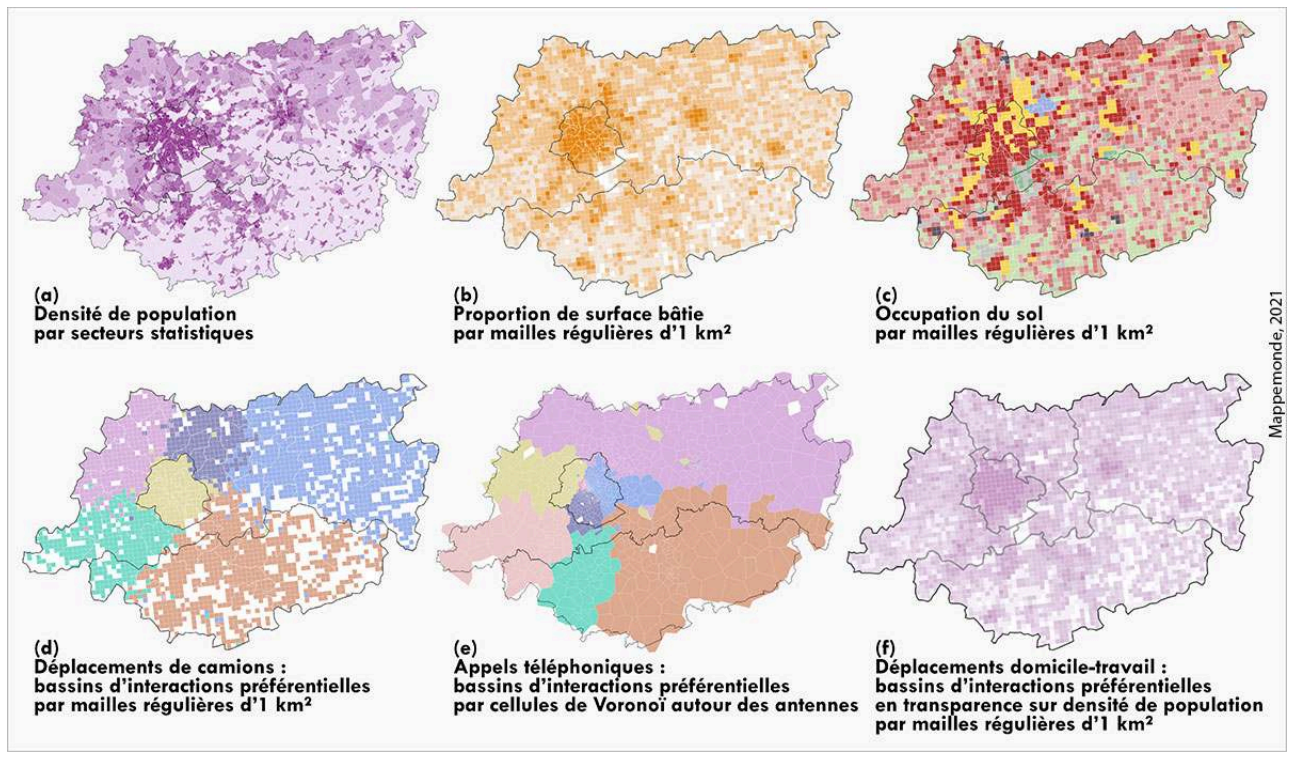

5 La composition de l'interface est avant tout centrée sur une carte qui occupe la majeure partie de l'écran (figure 2). On notera que même si la géovisualisation est conçue en responsive design (s'adaptant à la taille de l'écran), sa consultation sur ordinateur reste bien plus commode que sur tablette ou téléphone au vu de la quantité d'informations potentiellement affichées. Ajoutons ici que la totalité du site est en langue anglaise afin d'éviter l'épineux problème de la traduction dans les trois langues officielles en Belgique (français, néerlandais et allemand).

6 Au lancement de l'outil, une première carte est générée sans choix explicite de la part de l'utilisateurtrice. Il ou elle peut ensuite interagir avec la carte affichée en (dé)zoomant, en s'y déplaçant, ou encore en cliquant sur une unité spatiale pour obtenir des éléments descriptifs supplémentaires dans un encart spécifique. La carte est accompagnée de son titre et de sa légende à gauche de l'interface de l'outil. La partie droite est dédiée à l'ensemble des menus permettant à l'utilisateur.trice de passer d'une carte à une autre. Trois menus généraux sont affichés, quelle que soit la carte à l'écran: le menu «dataset» permettant de choisir un jeu de données à représenter; le menu «study area " pour afficher la même carte à une autre échelle (nationale si la carte affichée l'est à l'échelle métropolitaine, et inversement) - si toutefois le jeu de données est disponible à l'échelle demandée. De la même façon, le menu «resolution level » permet de faire apparaître la même carte à un niveau de granularité différent. Cette structure en différents menus permet de naviguer entre les échelles ou entre les variantes d'une même carte sans avoir à revenir systématiquement au sommet d'une arborescence unique. Vérifier qu'un phénomène constaté à une échelle et à une granularité données s'observe également à une autre échelle ou à une autre granularité est ainsi rendu particulièrement aisé.

Ces trois menus généraux sont, selon les cas, accompagnés d'un menu secondaire propre au jeu de données sélectionné. En particulier, si la carte affichée est une partition en bassins d'interactions préférentielles comme c'est le cas dans la figure 2, ce menu permet d'accéder à toutes les partitions opérées pour ce même jeu de données, en un nombre variable de bassins. Ce menu secondaire permet aussi de gérer la 
superposition des communautés aux structures démographiques, morphologiques et économiques. Enfin, selon les cas, des raccourcis sont générés pour simplifier la navigation entre les différentes variantes d'une même partition de l'espace en bassins d'interactions préférentielles.

Figure 2. Aperçu commenté de l'outil de géovisualisation du côté utilisateur

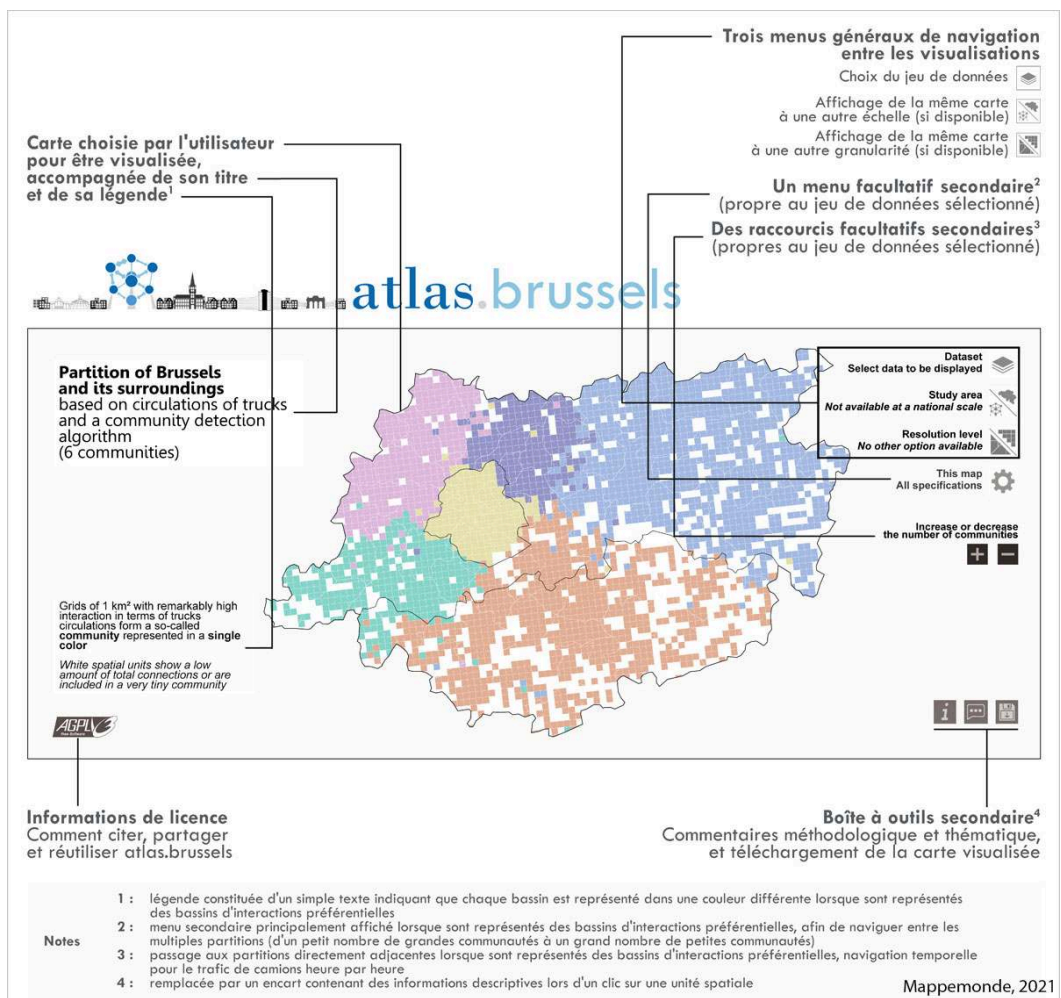

Le bas de l'écran est dédié à des informations annexes. En bas à droite, une boîte à outils secondaire permet d'obtenir des informations supplémentaires sur la construction de la carte affichée et des éléments de lecture ; un bouton permet aussi son téléchargement en image vectorielle (svg) ou matricielle (png). Cette boîte à outils est remplacée, lors d'un clic sur une unité spatiale, par ses informations attributaires. En bas à gauche, un bouton rappelle les informations de licence et les règles de citation, de partage et de réutilisation d'atlas.brussels.

L'utilisateur-trice est ainsi assez libre de naviguer entre les cartes implémentées dans l'outil, en n'étant pas nécessairement guidé selon un parcours-type. Le choix opéré dans le développement d'atlas.brussels est justement de présenter la quasi-totalité des résultats sans les éditorialiser outre mesure, comme cela pourrait être le cas dans les publications scientifiques au format plus contraint, ou dans des géovisualisations de type story maps. À ce titre, s'il fallait situer cette géovisualisation dans le cube de MacEachren qui fait référence (MacEachren, 1994 ; MacEachren et al., 2004 ; Davoine et al., 2014), atlas.brussels se trouverait probablement au cœur du cube (figure 3); son positionnement est, en effet, relativement intermédiaire sur chacune des trois dimensions qui le composent (degré d'interactivité, public cible et finalité). 
Figure 3. Positionnement d'atlas.brussels dans les stratégies de géovisualisation selon le public cible, la finalité et le degré d'interactivité

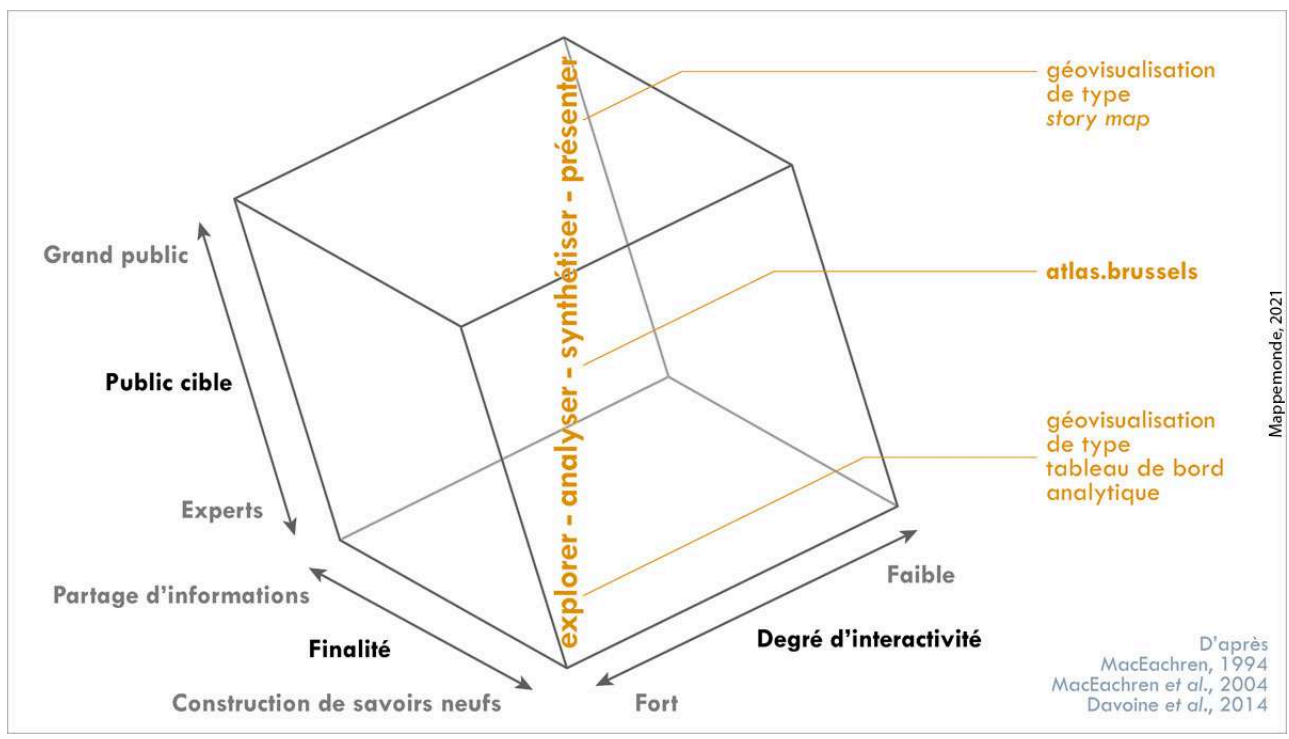

atlas.brussels ne se situe pas davantage à la base du cube de MacEachren (interactivité forte, public d'experts, construction de savoirs neufs), car les cartes visualisables, même si elles sont nombreuses, sont toutes explicitement implémentées dans l'outil. À titre d'exemple, seules les partitions des réseaux d'interactions en bassins d'interactions préférentielles calculées en amont sont disponibles. Ces partitions ont été réalisées sur six jeux de données différents et leurs variantes, certaines de ces données étant dites non conventionnelles. Ces partitions sont présentées aux côtés de cartes de contextualisation et d'analyses complémentaires (figure 4). Elles sont une manière de révéler la structure des réseaux et ce que ces réseaux disent des territoires, même si atlas.brussels ne montre pas explicitement ces réseaux. La section suivante détaille l'approche qui a mené à ces partitions de l'espace en bassins d'interactions préférentielles et les implications en termes de sémiologie. 
Figure 4. Types de cartes disponibles dans atlas.brussels selon le jeu de données, l'échelle, la granularité et des fonctionnalités supplémentaires disponibles

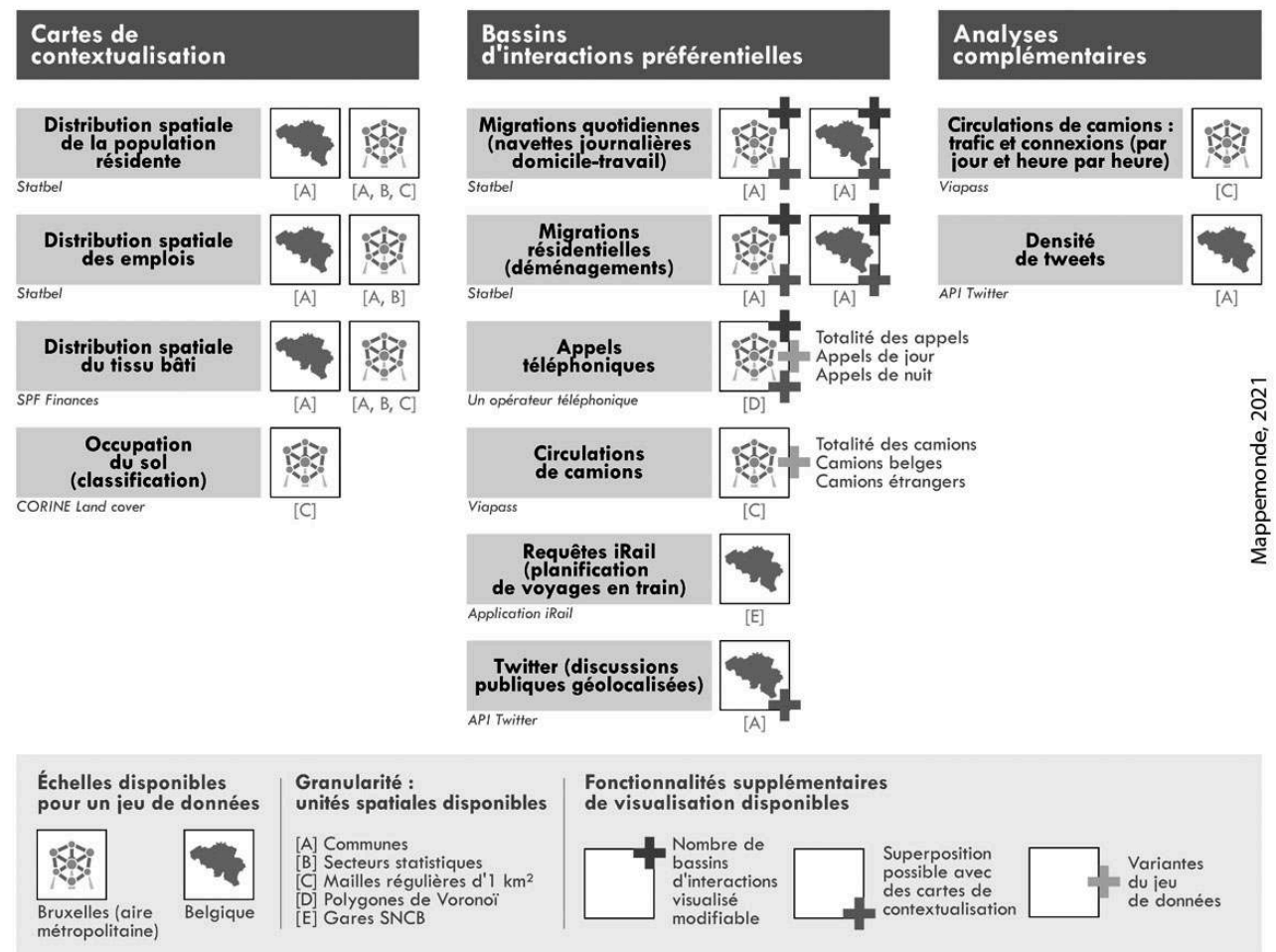

\section{Une méthode principale pour synthétiser et comparer de multiples réseaux d'interaction : la détection de communautés}

La visualisation de réseaux d'interaction peut s'avérer difficile, non seulement lorsqu'il s'agit d'en extraire des éléments synthétiques, mais aussi lorsqu'il s'agit d'établir des comparaisons entre réseaux. L'approche utilisée au cours du projet «Bru-Net » a été de mobiliser systématiquement des méthodes de détection de communautés, en particulier la méthode dite de Louvain, afin de détecter dans chacun des réseaux prenant la forme de matrices d'interaction, des unités spatiales qui s'avèrent être en interrelation significativement plus conséquente entre elles qu'avec les autres (Reichardt et Bornholdt, 2004 ; Blondel et al., 2008, 2010 ; Adam et al., 2018a ; Adam, 2019). Ceci a, dans un premier temps, reposé sur l'obtention, la préparation, la critique et le nettoyage de données spatialisées reliant deux à deux des unités spatiales. Dans un deuxième temps, le partitionnement du territoire en bassins d'interactions préférentielles a été effectué à la fois à l'échelle nationale et à l'échelle de l'aire métropolitaine bruxelloise. Il résulte de l'exploitation de ces algorithmes de partitionnement des territoires, des communautés ou des bassins, terme qui est préféré ici et d'autant plus justifié que les communautés mathématiques détectées s'avèrent dans la plupart des cas contiguës. La même méthode de détection de communautés a ainsi été exploitée pour l'ensemble des réseaux d'interaction, afin de rendre comparables et visualisables les résultats sous forme de cartes chorochromatiques. Cette méthode de représentation permet de montrer une facette notable de la structure 
des réseaux, tout en évitant de représenter les flux en tant que tels qui ne peuvent pas l'être pour certains pour des questions de confidentialité des données brutes et qui conduisent à une carte illisible tellement les lignes sont enchevêtrées (Renault, 1987 ; Cauvin et Reymond, 1991). Ceci permet alors d'analyser visuellement l'organisation spatiale en bassins ainsi détectés, et comment ils peuvent être comparés à d'autres éléments de différenciation de l'espace, notamment démographiques, morphologiques et socio-économiques.

Dans son implémentation de base, la méthode de Louvain établit une partition optimale du territoire d'étude en un nombre unique de bassins par maximisation de la modularité, une grandeur rapportant les liens internes aux communautés aux liens entre communautés. Une variante de la méthode mobilisée ici permet malgré tout de faire varier le nombre de bassins détectés, d'un petit nombre de grands bassins à un grand nombre de petits (pour plus d'information sur les principes de la méthode et sur sa variante, voir Reichardt et Bornholdt, 2004 ; Blondel et al., 2008, 2010 ; Adam et al., 2018a; Adam, 2019). Ces bassins sont ici générés à deux échelles pour accroître la connaissance sur l'extension spatiale de Bruxelles et sa fragmentation interne (figure 5). À l'échelle nationale, la détection de ces bassins permet d'identifier l'extension spatiale des bassins, pour la plupart centrés sur les grandes villes belges. Leur extension dépend de la nature des données utilisées, et ce particulièrement pour Bruxelles qui nous intéresse ici (en jaune au centre des cartes à l'échelle nationale) : les bordures de l'aire métropolitaine bruxelloise dépendent tant de la nature des données que du jeu de paramètres associés à la méthode. À l'échelle intra-métropolitaine, l'apport majeur est l'identification de bassins cohérents et donc de lignes (plus ou moins marquées) de fragmentation internes.

Ces analyses ont premièrement montré qu'à l'échelle nationale, les interactions ont lieu avant tout au sein des provinces administratives; la frontière linguistique entre la Flandre néerlandophone et la Wallonie francophone est encore moins perméable que les limites provinciales et elle joue un rôle de frein majeur dans les interactions. Deuxièmement, il y a une inadéquation majeure entre les limites de la région BruxellesCapitale, une des entités régionales politico-administratives à l'autonomie très large formant la Belgique au même titre que la Flandre et la Wallonie, et l'extension spatiale réelle de l'agglomération urbaine. Ceci est révélé non seulement par des éléments de contextualisation démographiques, morphologiques et économiques, mais aussi de manière plus inédite par les divers types de bassins d'interactions préférentielles détectés. Notons enfin qu'à l'échelle intra-urbaine, les bassins détectés prennent, pour la plupart des jeux de données, la forme d'une organisation sectorielle à la Hoyt, en «parts de tarte» structurées par rapport au Pentagone, le centre historique de Bruxelles. 
Figure 5. Six aperçus de cartes chorochromatiques pouvant être générées indiquant les bassins d'interactions préférentielles détectés à partir des déménagements selon l'échelle choisie et la finesse de la partition

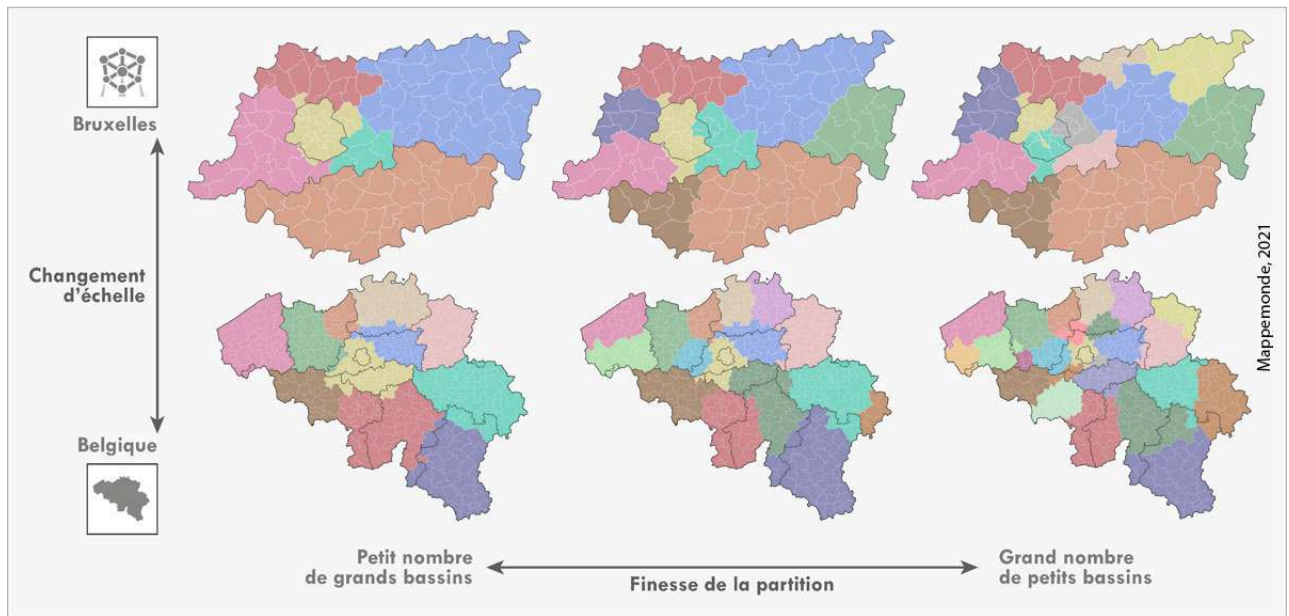

De ce projet de recherche ont découlé diverses valorisations, avant tout sous forme d'articles scientifiques publiés dans des revues à comité de lecture, bien souvent focalisés sur un seul ou sur un nombre restreint de jeux de données. Ce relatif morcellement des résultats est une des raisons du développement d'atlas.brussels, qui répond plus largement à quatre objectifs : rassembler, compléter, étendre et valoriser ces résultats.

\section{Les quatre objectifs d'atlas.brussels : rassembler, compléter, étendre, valoriser}

Le premier objectif de l'outil atlas.brussels est tout d'abord de rassembler les travaux qui peuvent donner, au travers des seules publications, une image morcelée des résultats du projet. Le schéma général « 1 jeu de données $=1$ article » a abouti à la détection de bassins de mobilité dans lesquels les habitantees ont significativement tendance à résider et à travailler à la fois, ou dans lesquels ils ou elles ont tendance à déménager significativement plus; de bassins d'interactions interpersonnelles, dans lesquels les appels téléphoniques ou les échanges sur les réseaux sociaux sont significativement plus marqués; de bassins économiques, dans lesquels les échanges de marchandises par le réseau de fret routier sont plus intenses ; etc. (Jones et al., 2016 ; Thomas et al., 2017 ; Adam et al., 2017, 2018a, 2018b; Decuyper et al., 2018; Finance et al., 2019a, 2019b). Autant de réseaux qui révèlent comment les interrelations entre acteurs de la ville (et ainsi entre portions de la ville) la forgent à diverses échelles. La comparaison entre les bassins détectés, par exemple, à partir des données de téléphonie mobile et à partir de données plus conventionnelles de déplacement domicile-travail peut être fastidieuse pour le lecteur ou la lectrice s'il s'agit de consulter plusieurs sources textuelles. atlas.brussels permet ainsi une comparaison bien plus aisée des résultats cartographiques construits sur les différents jeux de données d'interaction.

Deuxièmement, atlas.brussels vient compléter chacun de ces articles en présentant l'ensemble des partitionnements réalisés. En effet, les articles se focalisent généralement sur le ou les quelques partitionnements qui s'avéraient les plus appropriés, à dires d'expert·es, pour un jeu de données et pour l'objectif de l'article. À 
l'inverse, atlas.brussels présente tous les partitionnements en un nombre varié de bassins réalisés à partir de la variante de la méthode de Louvain. Six découpages distincts de l'espace sont, par exemple, proposés pour les déplacements domiciletravail à l'échelle nationale, soit de 6 grands à 26 petits bassins. De la sorte, l'utilisateur.trice peut juger non seulement de la représentation qui lui semble la plus pertinente, mais également de l'imbrication quasiment hiérarchique des petits bassins dans des bassins plus larges.

17 À cette occasion, profitant des possibilités offertes par l'interactivité de l'outil, de nouvelles fonctionnalités ont été intégrées afin d'étendre la visualisation à la comparaison visuelle entre bassins dessinés par détection de communautés et structures morphologiques (densité de bâti), démographiques (densité de population), ou économiques (densité d'emplois). Les bassins, par défaut représentés sous forme de cartes chrorochromatiques, peuvent être visualisés par leurs seuls contours en superposition de ces autres éléments comme dans la figure 1f. Le survol d'une communauté permet de la mettre en exergue afin de cerner précisément son emprise spatiale et la structure morphologique, démographique ou économique en arrière-plan. Le quatrième objectif de l'outil est d'ouvrir les résultats de recherche au-delà des publics-cibles traditionnels des publications scientifiques, dans une démarche de valorisation de la recherche. L'accès difficile aux publications (en raison du prix ou du format parfois obscur pour qui n'est pas dans le domaine) pour diverses composantes de la société civile pouvant malgré tout avoir un certain intérêt pour les questionnements développés est ici levé en proposant cette visualisation ouverte à tous.

19 Enfin, l'utilisation d'outils libres dans son développement a été jugée primordiale; les aspects techniques de son développement sont présentés ci-après.

\section{La géovisualisation côté développement}

L'interface de l'outil de géovisualisation a été développée en utilisant les langages HTML, CSS et JavaScript en suivant une structure relativement similaire à celle du Mobiliscope (Lecomte et al., 2018). Dans une étape préalable, les partitions en bassins d'interactions préférentielles représentées dans l'outil de géovisualisation ont - pour la quasi-totalité d'entre elles - été réalisées par Arnaud Adam dans le cadre de sa thèse de doctorat (Adam, 2019). Les fichiers de sortie de ces analyses (appartenance de chaque unité spatiale à telle communauté) ont d'abord été retravaillés, joints à des shapefiles et convertis sur le logiciel Qgis en format geojson afin de pouvoir être ensuite appelés dans l'outil de géovisualisation.

La page est d'abord structurée via un code HTML général qui produit notamment les différents menus permettant la navigation entre les cartes proposées (partie gauche de la figure 6). Ce code fait appel à des scripts supplémentaires stockés dans des fichiers externes (figure 6), notamment un fichier CSS pour gérer le style des éléments affichés, d3.js pour la génération des cartes (Bostock et al., 2011 ; Bostock, 2016) et jquery.js qui gère l'interactivité de la page. L'utilisation de jquery est habituelle; celle de $d 3$.js, qui n'est pas une bibliothèque de fonctions cartographiques stricto sensu comme l'est, par exemple, Leaflet, s'explique par la volonté de conserver une très grande latitude dans 
les développements ultérieurs de l'outil, afin de coupler, par exemple, des visualisations cartographiques et graphiques.

Outre d3.js et jquery, le script JavaScript qui permet la génération des cartes, leur interactivité et celle des menus, est scindé en deux parties. Une première partie, dite générique, correspond aux scripts nécessaires à la génération de n'importe quelle carte. La seconde, dite spécifique, est externalisée dans un autre fichier et comprend une fonction distincte pour chaque carte pouvant être générée, où figurent le chemin d'accès du jeu de données nécessaire à l'affichage de la carte demandée, son mode de représentation, ou encore les éléments d'habillage.

Ainsi, lorsque l'utilisateurtrice clique sur un bouton du menu pour changer de carte, c'est la combinaison de divers de ces scripts qui va permettre la mise à jour de la page web. La bibliothèque $\mathrm{d} 3$.js, appelée pour ses fonctions basiques de visualisation (carto)graphique, est alors couplée au script JavaScript générique propre à atlas.brussels, valable pour toutes les cartes, et au script JavaScript spécifique propre à la carte demandée ; le code CSS vient, en sus, gérer le style d'affichage (figure 6). Grâce à cette structure séparant les fonctions génériques des fonctions spécifiques, la génération de cartes à plusieurs échelles et à plusieurs granularités est facilitée et, en partie au moins, optimisée.

Figure 6. Structure de l'outil de visualisation côté développement et combinaison schématique des scripts lors de la génération d'une nouvelle carte

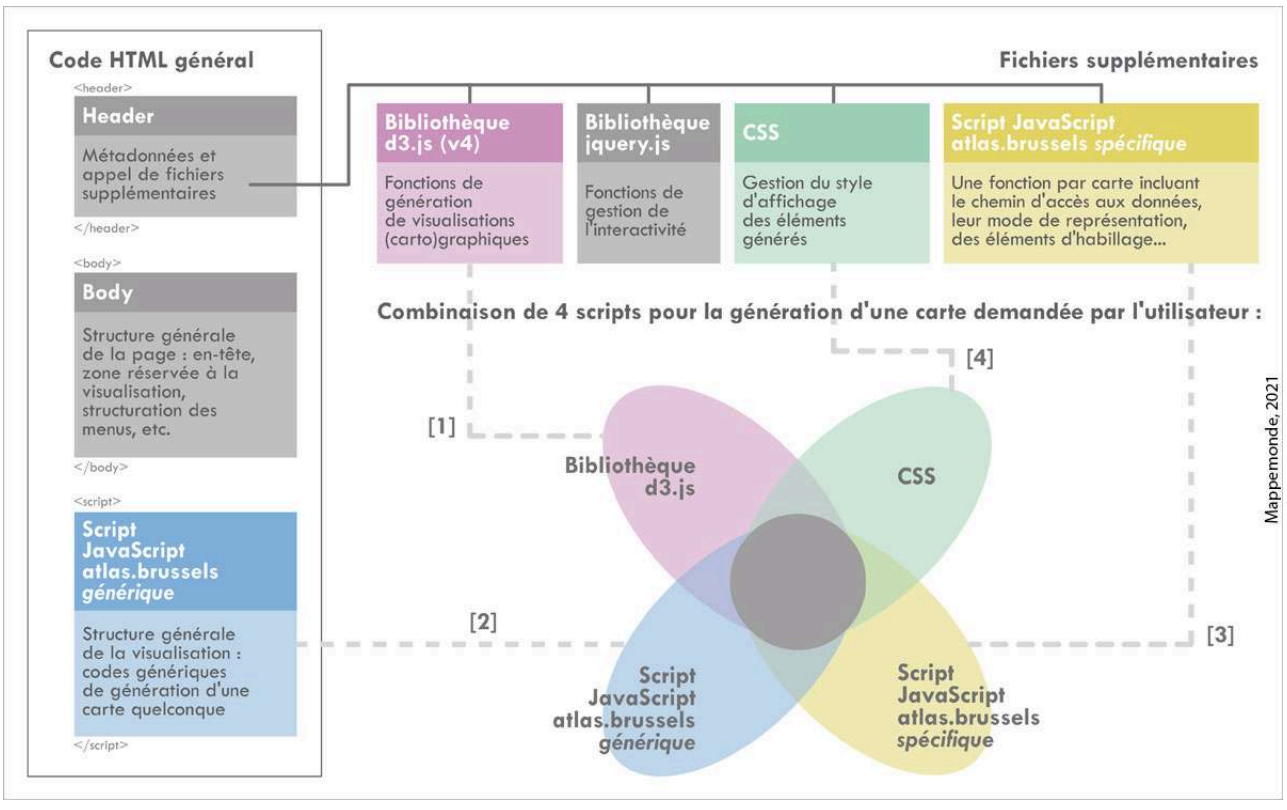

La totalité de ce code peut être consultée, téléchargée et réutilisée selon les termes de la licence AGPL (licence libre copyleft) impliquant le partage, sous les mêmes termes, des modifications opérées par l'utilisateur.trice.

\section{atlas.brussels : un outil de diagnostic et de valorisation de travaux scientifiques sur Bruxelles}

atlas.brussels constitue ainsi non seulement un outil de géovisualisation interactif innovant de par l'approche développée dans sa construction, mais aussi un outil 
destiné à des publics divers et notamment au grand public, à la société civile et aux politiques. Ces derniers peuvent s'en servir comme outil de diagnostic de l'extension spatiale bruxelloise à travers diverses dimensions, pour vérifier l'inadéquation affirmée de longue date entre limites politico-administratives et réalité de cette extension. Ils peuvent également s'en servir comme outil de diagnostic de la fragmentation interne de la métropole, un enjeu politique majeur quel que soit le contexte géographique. Analyser la fragmentation spatiale de Bruxelles à partir de matrices d'interaction, aussi intéressant que cela puisse être pour des équipes de recherche, nécessite de mobiliser des outils spécifiques permettant de transmettre des résultats de manière compréhensible par le plus grand nombre. Les géovisualisations de ce type peuvent ainsi constituer un outil de dialogue fondamental entre la recherche académique et le politique, par la mise en exergue de la façon dont les habitantees et autres acteurs appréhendent et forgent leur territoire.

À l'avenir, il s'agirait, en cas de développement d'une version plus aboutie d'atlas.brussels, d'enrichir encore le contenu thématique avec de nouveaux résultats, éventuellement obtenus par d'autres équipes de recherche. Il pourrait s'agir également d'améliorer encore, pour l'utilisateur.trice, la comparaison des cartes générées par l'outil, en permettant, par exemple, la visualisation et la comparaison de deux cartes affichées côte à côte, en split screen. D'autres fonctionnalités développées, mais non exploitées, pourraient être implémentées dans le respect des questions de confidentialité, comme l'affichage des flux principaux, depuis ou vers une unité spatiale, lorsque sont affichés des bassins d'interactions préférentielles.

\section{Financement}

27 Le développement d'atlas.brussels a pris place dans le projet «Bru-Net: un nouveau regard sur les relations à l'intérieur de l'aire métropolitaine bruxelloise » porté par Isabelle Thomas et Jean-Charles Delvenne de 2015 à 2019. Ce projet a été financé par la région Bruxelles-Capitale via Innoviris, l'Institut bruxellois pour la Recherche et l'Innovation.

\section{BIBLIOGRAPHIE}

ADAM A. (2019). Exploring new geographies of interactions in and around the metropolitan area of Brussels. Thèse de doctorat de l'UCLouvain, Louvain-la-Neuve, Belgique. En ligne : https:// tel.archives-ouvertes.fr/tel-02284406/document

ADAM A., DELVENNE J.C. et THOMAS I. (2018a). "Detecting communities with the multi-scale Louvain method: robustness test on the metropolitan area of Brussels". Journal of Geographical systems, vol. $20, \mathrm{n}^{\circ}$ 4. DOI : 10.1007/s10109-018-0279-0 
ADAM A., CHARLIER J., DEBUISSON M., DUPREZ J.P., REGINSTER I. et THOMAS I. (2018b). « Bassins résidentiels en Belgique : deux méthodes, une réalité ? ». L'Espace géographique, t. 47, p. 35-50. DOI : 10.3917/ eg.471.0035

ADAM A., DELVENNE J.-C. et THOMAS I. (2017). “Cartography of interaction fields in and around Brussels: commuting, moves and telephone calls". Brussels Studies, n 118 . DOI : 10.4000/brussels. 1601

BLONDEL V., KRINGS G. et THOMAS I. (2010). "Regions and borders of mobile telephony in Belgium and in the Brussels metropolitan zone". Brussels Studies, n 42. DOI : 10.4000/brussels.806 BLONDEL V., GUILLAUME J.-L., LAMBIOTTE R. et LEFEBVRE É. (2008). “Fast unfloding of communities in large networks". Journal of Statistical Mechanics: Theory and Experiment, oct. 2008. DOI : 10.1088/1742-5468/2008/10/P10008 возтоск M. (2016). d3.js - Data-driven documents. En ligne : https://d3js.org BOSTOCK M., OGIEVETSKY V. et HEER J. (2011). "D³ data-driven documents". IEEE transactions on visualization and computer graphics, vol. 17, $\mathrm{n}^{\circ}$ 12. DOI : 10.1109/TVCG.2011.185 CAUVIN C. et REYMOND H. (1991). «Interaction spatiale et cartographie : les solutions de W. Tobler ». Espace Populations Sociétés, n 3, p. 467-485. DOI : 10.3406/espos.1991.1489 DAVOINE P.-A., MATHIAN H. et SAINT-MARC C. (2014). « Productions actuelles : quelles temporalités, quelle diversité ? "Séminaire PUCA. Représentations dynamiques des temporalités des territoires. La Défense, PUCA, Paris, France. En ligne : https://halshs.archives-ouvertes.fr/halshs-01368545 DECUYPER A., GANDICA Y., CLOQUET C., THOMAS I. et DELVENNE J.-C. (2018). “Measuring the effect of node aggregation on community detection". Working paper. En ligne : https://arxiv.org/abs/ 1809.08855

DUJARDIN C., THOMAS I. et TULKENS H. (2007). « Quelles frontières pour Bruxelles ? Une mise à jour ». Reflets et perspectives de la vie économique, $\mathrm{n}^{\circ}$ 2-3, t. XLVI, p. 155-176. DOI : 10.3917/rpve.462.0155

FINANCE O., ADAM A., JONES J. et THOMAS I. (2019a). « Révéler la polarisation économique d'une ville à partir de traces GPS de camions. Le cas de Liège ». Bulletin de la Société géographique de Liège, vol. $72, \mathrm{n}^{\circ} 1$. DOI : 10.25518/0770-7576.5816

FINANCE O., ADAM A. et THOMAS I. (2019b). « Circulations de camions en Belgique. Les big-data au service de la connaissance du territoire ». Poster proposé aux Rencontres de Théo Quant. En ligne : https://halshs.archives-ouvertes.fr/halshs-02005550/

GODIN M. et HINDRIKS J. (2015). « Disparités et convergence économiques : rattrapage économique wallon? » Regards économiques, n 120, 14 p. En ligne : https://www.regards-economiques.be/ images/reco-pdf/reco_155.pdf

JONES J., CLOQUET C., ADAM A., DECUYPER A. et THOMAS I. (2016). "Belgium through the lens of rail travel requests: does geography still matter? ". ISPRS International Journal of Geo-Information, vol. 5. DOI : 10.3390/ijgi5110216

JONES J., PEETERS D. et THOMAS I. (2015). “Is cities delineation a pre-requisite for urban modelling? The example of land price determinants in Brussels". Cybergeo: European Journal of Geography. DOI : 10.4000/cybergeo.26899

LeCOMTE C., VAllÉE J., LE ROUX G. et COMMENGes H. (2018). « Le Mobiliscope, un outil de géovisualisation des rythmes quotidiens des métropoles ». Mappemonde, 123. En ligne : https:// journals.openedition.org/mappemonde/522 
MACEACHREN A. M. (1994). “Visualization in Modern Cartography: Setting the Agenda”. In MACEACHREN A. M. et TAYLOR D. R. F. (éd.), Visualization in Modern Cartography, p. 1-12, Oxford: Pergamon.

MACEACHREN A. M., GAHEGAN M., PIKE W., BREWER I., CAI G., LENGERICH E. et HARDISTY F. (2004).

"Geovisualization for Knowledge Construction and Decision Support". IEEE Computer Graphics and Applications, vol. 24, nº 1, p. 13-17. DOI : 10.1109/MCG.2004.1255801

REICHARDT J. et BORNHOLDT S. (2004). "Detecting fuzzy community structures in complex networks with a Potts Model". Physical Review Letters, vol. 93, n² 21. DOI : 10.1103/PhysRevLett.93.218701

RENAULT E. (1987). « Représentation des flux en CAO ». Mappemonde, nº 87-3. En ligne : http:// www.mgm.fr/PUB/Mappemonde/M387/p42-46.pdf

STRALE M. (2019). « Les déplacements entre Bruxelles et sa périphérie : des situations contrastées ». Brussels Studies, $n^{\circ}$ 137. DOI : https://doi.org/10.4000/brussels.2831

THOMAS I., COTTEELS C., JONES J. et PEETERS D. (2012). "Revisiting the extension of the Brussels urban agglomeration: new methods, new data... new results?" Belgeo, ${ }^{\circ} 1-2$. DOI : 10.4000/belgeo.6074 THOMAS I., ADAM A. et VERHETSEL A. (2017). “Migration and commuting interaction fields: A new geography with a community detection algorithm?" Belgeo, $n^{\circ} 4$. DOI : 10.4000/belgeo.20507 VANDERSTRAETEN L. et VAN HECKE É. (2019). «Les régions urbaines en Belgique ». Belgeo, $\mathrm{n}^{\circ}$ 1. DOI : $10.4000 /$ belgeo. 32246

VAN HECKE É. (1999). «Le réseau urbain en Belgique : dynamiques fonctionnelles et flux ». Hommes et Terres du Nord, $\mathrm{n}^{\circ}$ 3, p. 166-175. DOI : 10.3406/htn.1999.2686

VAN WYNSBERGHE C. (2011). L'exemple belge. Les faiblesses de la formule fédérale mise en place en Belgique. Rapport pour le Réseau québécois de réflexion sur le fédéralisme. En ligne : https://uclouvain.be/ cps/ucl/doc/spri/documents/

Etude_sur_le_federalisme_belge_C._Van_Wynsberghe_(francais).pdf

VAN WYNSBERGHE C., POIRIER J., SINARDET D. et TULKENS F. (2009). « Le développement politique et institutionnel de la Zone métropolitaine de Bruxelles : constats et perspectives ». Brussels Studies. DOI : $10.4000 /$ brussels.958

\section{RÉSUMÉS}

atlas.brussels est un outil de géovisualisation interactive en ligne qui permet de questionner l'extension spatiale et la fragmentation interne de l'aire métropolitaine bruxelloise. Pour ce faire, l'outil se focalise de manière originale sur des réseaux d'interaction spatiale appréhendés à la fois à l'échelle nationale et métropolitaine. Parmi les diverses composantes considérées figurent les déplacements de personnes (notamment domicile-travail), les communications (notamment les appels téléphoniques) ou encore les échanges économiques (notamment par le fret routier). atlas.brussels permet pour cela de rassembler, compléter, étendre et valoriser des travaux de recherche sur l'aire métropolitaine bruxelloise, et reste ouvert à l'intégration d'autres jeux de données.

atlas.brussels is an interactive online geovisualization tool that questions the spatial extension and the internal fragmentation of the Brussels metropolitan area. To do this, the tool focuses in an original way on spatial interaction networks considered at both the national and the metropolitan scales. Among these networks appear movements of people (as home-to-work 
commuting), communications (as phone calls) and economic interactions (through the road freight interactions). Therefore, atlas.brussels combines, supplements, expands and promotes researches made on the metropolitan area of Brussels, and remains open to the integration of other datasets.

\section{INDEX}

Keywords : geovisualisation, interactive cartography, Brussels, community detection, d3.js Mots-clés : géovisualisation, cartographie interactive, Bruxelles, détection de communautés, d3.js

\section{AUTEURS}

\section{OLIVIER FINANCE}

Université de Strasbourg, Laboratoire Image, Ville, Environnement

\section{ARNAUD ADAM}

Université catholique de Louvain, Louvain Institute of Data Analysis and Modeling in economics and statistics, Center for Operations Research and Econometrics

\section{ISABELLE THOMAS}

Université catholique de Louvain, Louvain Institute of Data Analysis and Modeling in economics and statistics, Center for Operations Research and Econometrics 